\title{
Effect of siRNA-mediated gene silencing of transketolase on A549 lung cancer cells
}

\author{
HUAN LU and HUILI ZHU \\ Department of Respiratory Medicine, Huadong Hospital, Fudan University, Shanghai 200040, P.R. China
}

Received October 29, 2015; Accepted May 4, 2017

DOI: $10.3892 / \mathrm{ol} .2017 .6916$

\begin{abstract}
The aim of the present study was to investigate the effects of transketolase (TKT) on cell proliferation, cell migration and interaction with other metabolism-associated genes in A549 lung cancer cells. A549 cells were transfected with three $T K T$-specific small interfering (si)RNAs, screened for the optimal transfection concentration, and sequenced with flow cytometry and reverse transcription-quantitative polymerase chain reaction (RT-qPCR). Cell viability was evaluated using Cell Counting Kit-8 (CCK-8), cell cycle was assessed by flow cytometric analysis. Cell migration was determined by scratch-wound and Transwell chamber assays. The changes in mRNA expression levels of glucose-6-phosphate dehydrogenase $(G 6 P D H)$, transaldolase $(T A L)$, sorbitol dehydrogenase (SORD), phosphoribosyl pyrophosphate synthetase 1 (PRPS1) and hexokinase $1(H K 1)$ were detected by RT-qPCR. siRNA-C at $50 \mathrm{nmol} / 1$ was selected for the subsequent experiments. Compared with the negative control, cell proliferation of the TKT-siRNA-C group was inhibited dramatically (CCK-8 $24 \mathrm{~h}$, $0.2984 \pm 0.0371$ vs. $0.0952 \pm 0.0063 ; \mathrm{P}<0.0001)$, the cell cycle was arrested at the $\mathrm{G}_{1} / \mathrm{G}_{0}$ cell cycle phase $(58 \pm 2.0 \%$ vs. $70 \pm 2.5 \%$; $\mathrm{P}=0.002)$, and cell migration ability was decreased [wound size, $254.71 \pm 34.96$ vs. $349.12 \pm 37.43 \mu \mathrm{m}(\mathrm{P}=0.0001)$; Transwell migration, $250 \pm 47.8 /$ field vs. $150 \pm 49.0 /$ field $(\mathrm{P}<0.0001)]$. The mRNA expression levels of G6PDH, TAL, SORD, PRPS1 and $H K 1$ were downregulated in the $T K T$-siRNA-C group compared with the negative control. The present study revealed that synthetic $T K T$-siRNA can inhibit A549 cell viability and migration, which may be due to arrest of the cell cycle and downregulation of relevant metabolic enzymes.
\end{abstract}

Correspondence to: Dr Huili Zhu, Department of Respiratory Medicine, Huadong Hospital, Fudan University, 221 Yan'an West Road, Shanghai 200040, P.R. China

E-mail: zhuhuili999@126.com

Key words: transketolase, small interfering RNA, lung cancer, A549 cells, transketolase-like protein 1

\section{Introduction}

An estimated 1.8 million new lung cancer cases occurred in 2012, accounting for $\sim 13 \%$ of total cancer diagnoses (1). During this time period, lung cancer was the most frequently diagnosed type of cancer and the leading cause of cancer-associated mortality among males; among females, it was the leading cause of cancer-associated mortality in more developed countries and the second leading cause of cancer-associated mortality in less developed countries (1). In 2011, lung cancer exhibited the highest morbidity and mortality rates among all cancer types in China (2).

Abundant supplies of resources and energy are essential for malignant proliferation. The pentose phosphate pathway, the only pathway for de novo ribose synthesis, has been identified as a tumor therapeutic target (3-5). The pentose phosphate pathway consists of an oxidative pathway and a non-oxidative pathway (6). In the oxidative pathway, by oxidative decarboxylation, glucose 6-phosphate is irreversibly converted into ribose 5-phosphate used for the synthesis of nucleotides, and two NADPH molecules are produced as reducing equivalents (6). The non-oxidative pathway joins the pentose phosphate pathway to glycolysis, and spare ribose or pentose molecules are reversibly transformed into glyceraldehyde 3-phosphate and fructose 6-phosphate by transketolase (TKT) and transaldolase (TAL), without restriction of the irreversible oxidative signaling pathway (6). A previous study revealed the upregulation of TKT activity in various types of cancer in vivo and in vitro (3). Our previous study demonstrated that oxythiamine exerts its anticancer effect via the inhibition of thiamine-dependent enzymes, particularly TKT (7).

RNA interference (RNAi) technology provides an advantageous platform for investigating the function of a given gene. Prior to RNAi technology, gene knockout was the main research tool in reverse genetics. However, RNAi technology, using siRNA or short hairpin RNA expression vectors to knockdown target genes in a sequence-specific way, may be more time-efficient, economic and convenient and is therefore an attractive tool for gene function exploration, particularly within cancer research fields (8). In the present study, siRNA vectors were applied with cationic liposomes to investigate the influence of the TKT gene on the metabolism of A549 cells. 


\section{Materials and methods}

Reagents and equipment. The following reagents and equipment were used in the present study: Gibco fetal bovine serum (FBS; Thermo Fisher Scientific, Inc., Waltham, MA, USA); Dulbecco's modified Eagle's medium (DMEM; Genom Biotech Pvt., Ltd., Mumbai, India); three types of TKT-specific siRNA duplex sequences and negative-control siRNA duplex sequence (catalog no. A10001) and transfection reagents (siRNA-Mate; catalog no. G04003; Shanghai GenePharma Co., Ltd., Shanghai, China); polymerase chain reaction (PCR) primers, as presented in Table I (Shanghai Sangon Pharmaceutical Co., Ltd., Shanghai, China); quantitative (q)PCR reaction kit (UltraSYBR Mixture; catalog no. CW0957; CWBiotech Co. Ltd., Beijing, China) and cell cycle detection kit (catalog no. CW2575S, CWBiotech Co. Ltd.); Cell Counting Kit-8 (CCK-8; Beyotime Institute of Biotechnology, Haimen, China); Transwell chambers and cell culture dishes (Corning Incorporated, Corning, NY, USA); Mastercycler ep realplex cycler (Eppendorf, Hamburg, Germany); FACSCalibur flow cytometer (BD Biosciences, Franklin Lakes, NJ, USA); NanoDrop 2000c spectrophotometer (Thermo Fisher Scientific, Inc.).

Cell culture. The A549 lung cancer cell line was provided by Zhongshan Hospital Central Laboratory of Fudan University (Shanghai, China). A549 cells were cultured in DMEM supplemented with $10 \%$ FBS, penicillin $(100 \mathrm{kU} / \mathrm{l})$ and streptomycin $(100 \mathrm{kU} / \mathrm{l})$ in a humidified incubator at $37^{\circ} \mathrm{C}$ and $5 \% \mathrm{CO}_{2}$.

siRNAs. The sequences of the siRNAs used in the present study were as follows: TKT-siRNA-A sense, 5'-CCGGCAAAUACU UCGACAATT-3'; antisense, 5'-UUGUCGAAGUAUUUG CCGGTT-3'. TKT-siRNA-B sense, 5'-GCAUCUAUAAGC UGGACAATT-3'; antisense, 5'-UUGUCCAGCUUAUAG AUGCTT-3'. TKT-siRNA-C sense, 5'-CCAGCCAACAGC CAUCAUUTT-3'; antisense, 5'-AAUGAUGGCUGUUGG CUGGTT-3'. Negative control siRNA and fluorescein amidite (FAM)-negative control siRNA sense, 5'-UUCUCCGAACGU GUCACGUTT-3'; antisense, 5'-ACGUGACACGUUCGG AGAATT-3'.

Transfection. FAM-labelled siRNA $(0,25,50$ and $75 \mathrm{nM})$ was transfected into A549 cells to determine the optimal transfection concentration. Cells were transfected with siRNAs when cell confluence $30-50 \%$, according to the manufacturer's protocol. FAM-siRNA $(25 \mu \mathrm{M} ; 0,2.2,4.4$ and $6.6 \mu \mathrm{l}$ ) was added into $200 \mu \mathrm{l}$ DMEM solution for $5 \mathrm{~min}$ and, subsequently, $8 \mu 1$ siRNA-mate was mixed into the solution for $30 \mathrm{~min}$. During this time, siRNA-siRNA-mate complexes were generated. During this procedure, cell culture medium was replaced with $2 \mathrm{ml}$ DMEM with serum (Gibco; Thermo Fisher Scientific, Inc.). The siRNA-siRNA-mate complexes were added to the cells and incubated for $6-8 \mathrm{~h}$ at $37^{\circ} \mathrm{C}$. Cells were digested with trypsin (Genom Biotech Pvt., Ltd.) for $2 \mathrm{~min}$ at room temperature, centrifuged at 1,000 $\mathrm{x}$ g for $5 \mathrm{~min}$ at room temperature and washed with ice-cold PBS (Genom Biotech Pvt., Ltd.) twice at room temperature. For each cell suspension, $\geq 10,000$ events were analyzed using a FACSCalibur flow cytometer and FlowJo software (version
7.6.1; Tree Star, Inc., Ashland, OR, USA) for data analysis. To determine the most effective transfection sequence, six groups were assessed and compared: Blank control (DMEM only), mock control (DMEM and siRNA mate), negative control (negative control siRNA), TKT-siRNA-A, TKT-siRNA-B and TKT-siRNA-C. Reverse transcription (RT-)qPCR was used to determine the TKT mRNA expression levels.

Reverse transcription $(R T)-q P C R$. Total RNA was extracted from the cells using TRIzol ${ }^{\circledR}$ reagent (CWBiotech Co. Ltd.). The quantity and integrity of mRNA was validated using a NanoDrop 2000c spectrophotometer (Thermo Fisher Scientific, Inc.) and gDNA Eraser was used to erase the genomic DNA at $42^{\circ} \mathrm{C}$ for $2 \mathrm{~min}$. Subsequently, HiFiScript cDNA Synthesis kit (catalog no. CW2569; CWBiotech Co. Ltd.) was used to reverse transcribe $1 \mu \mathrm{g}$ total RNA at $37^{\circ} \mathrm{C}$ for $15 \mathrm{~min}$ and $85^{\circ} \mathrm{C}$ for $5 \mathrm{sec}$ into cDNA. qPCR was performed using a Mastercycler ep realplex cycler with SYBR ${ }^{\circledR}$ Green Master Mix (catalog no. CW0957; CWBiotech Co. Ltd.). Primers for TKT, glucose-6-phosphate dehydrogenase (G6PDH), TAL, phosphoribosyl pyrophosphate synthetase 1 (PRPS1), sorbitol dehydrogenase (SORD), hexokinase 1 (HK1), transketolase-like 1 (TKTL1), transketolase-like 2 (TKTL2) and $\beta$-actin were used, and the details are listed in Table I. The amplification procedure consisted of $95^{\circ} \mathrm{C}$ for $10 \mathrm{~min}$, followed by 40 cycles of $95^{\circ} \mathrm{C}$ for $15 \mathrm{sec}$ and $60^{\circ} \mathrm{C}$ for $60 \mathrm{sec}$. Each sample was performed in triplicate. The quantification cycle $(\mathrm{Cq})$ values of the target genes were normalized to the housekeeping gene $\beta$-actin, and relative expression levels were compared according to the $2-\Delta \Delta \mathrm{Cq}$ method (9).

CCK-8 assay. Following a 72-h transfection, cells were seeded into 96-well plates $(2,000$ cells/per well) and incubated for 24,48 and $72 \mathrm{~h}$ at $37^{\circ} \mathrm{C}$ in an atmosphere containing $5 \% \mathrm{CO}_{2}$. Following incubation, $10 \mu \mathrm{l}$ CCK-8 solution was added to the wells, according to the manufacturer's protocol. Following a further $2 \mathrm{~h}$ incubation, optical density (OD) was evaluated at $450 \mathrm{~nm}$, which provided an indirect indication of the number of viable cells.

Cell cycle analysis. Following 72 h transfection, cells (when confluence reached more than 90\%) were digested by trypsin (Genom Biotech Pvt., Ltd.) for 2 min at room temperature, centrifuged at $1,000 \mathrm{x} \mathrm{g}$ for $5 \mathrm{~min}$ at room temperature and washed with ice-cold PBS (Genom Biotech Pvt., Ltd.) twice at room temperature. Cells were fixed with $75 \%$ ethanol overnight at $4^{\circ} \mathrm{C}$ and subsequently dyed with propidium iodide for $30 \mathrm{~min}$ at $37^{\circ} \mathrm{C}$ in the dark. For each cell suspension, $\geq 10,000$ events were analyzed using a FACSCalibur flow cytometer and FlowJo software (version 7.6.1; Tree Star, Inc., Ashland, OR, USA) for data analysis.

Wound healing. Following $72 \mathrm{~h}$ transfection, $2 \times 10^{6}$ cells were seeded into 6-well plates. When the confluence reached $100 \%$, linear wounds were created using a sterile $10 \mu \mathrm{l}$ plastic pipette tip and serum-free medium was added. Following 0, 6, 12 and $24 \mathrm{~h}$ incubations at $37^{\circ} \mathrm{C}$ in an atmosphere containing $5 \%$ $\mathrm{CO}_{2}$, the wounds were imaged using an inverted microscope (Olympus, Japan) under bright-field illumination. ImageJ software (version $1.48 \mathrm{u}$; National Institutes of Health) was used to calculate the width of wounds. 
Table I. Polymerase chain reaction primers.

\begin{tabular}{|c|c|c|}
\hline Primer & Sequence (5' to $3^{\prime}$ ) & Product length \\
\hline \multirow[t]{2}{*}{$\beta$-actin } & F: TGACGTGGACATCCGCAAAG & $205 \mathrm{~kb}$ \\
\hline & R: CTGGAAGGTGGACAGCGAGG & \\
\hline \multirow[t]{2}{*}{ TKT } & F: TACCCAAGTGATGGCGTTGC & $129 \mathrm{~kb}$ \\
\hline & R: GACCTGGAAGTCCTCATTGTTGTTAT & \\
\hline \multirow[t]{2}{*}{ G6PDH } & F: TGTTGTCCCGGTTCCAGATG & 217 bp \\
\hline & R: TGCATGAGCCAGATAGGCTG & \\
\hline \multirow[t]{2}{*}{ TAL } & F: ATGGTGAGGAAGTCACAGCC & $201 \mathrm{bp}$ \\
\hline & R: ATTTGTTGGGCGCATCCTTG & \\
\hline \multirow[t]{2}{*}{ SORD } & F: AAGACCTCATTTGGGCCTGG & $92 \mathrm{bp}$ \\
\hline & R: AAGCCCAACAACCTTTCCCT & \\
\hline \multirow[t]{2}{*}{ HK1 } & F: TTATCACGCAGGCGGTTCA & 147 bp \\
\hline & R: TGCCAAAGAAATCCTGACCC & \\
\hline \multirow[t]{2}{*}{ PRPS1 } & F: TGCGAGAAATAGCAGGACCG & $263 \mathrm{bp}$ \\
\hline & R: CCAGGAGACCTGAGTGACCT & \\
\hline \multirow[t]{2}{*}{ TKTL1-1 } & F: ACTAATGGCGGATGCTGAGG & 153 bp \\
\hline & R: CATGATGTAGGGTGGCCGGA & \\
\hline \multirow[t]{2}{*}{ TKTL1-2 } & F: ATGGCTCGGACAAGGACTGG & $191 \mathrm{bp}$ \\
\hline & R: GGCGGTTCACATCAAAGATTGC & \\
\hline \multirow[t]{2}{*}{ TKTL1-3 } & F: AAACTTGCCCCGAGTCCAC & $125 \mathrm{bp}$ \\
\hline & R: GGTAAGGAATGCAGGGCTCA & \\
\hline \multirow[t]{2}{*}{ TKTL2 } & F: ATCCATTCCATCAGGGCCAC & $175 \mathrm{bp}$ \\
\hline & R: GGATAGGAGCAGCATGTCCC & \\
\hline
\end{tabular}

TKT, transketolase; G6PDH, glucose-6-phosphate dehydrogenase; TAL, transaldolase; SORD, sorbitol dehydrogenase; HK1, hexokinase; PRPS1, phosphoribosyl pyrophosphate synthetase 1; TKTL, transketolase-like; F, forward; R, reverse.

Transwell migration assay. Following a $72 \mathrm{~h}$ transfection, $1.0 \times 10^{5}$ cells in $100 \mu \mathrm{l}$ serum-free medium were added to the upper compartment of each Transwell chamber and $600 \mu \mathrm{l}$ DMEM supplemented with $10 \%$ FBS was added into each of the lower compartments. Following a $12 \mathrm{~h}$ incubation at $37^{\circ} \mathrm{C}$ in an atmosphere containing $5 \% \mathrm{CO}_{2}$, cells that had penetrated the membrane were dyed with $0.1 \%$ crystal violet for $20 \mathrm{~min}$. Five randomly selected fields of view were observed using a microscope (magnification, x10 and x20; DP72; Olympus, Japan) under bright-field illumination and cells that penetrated the Transwell chamber membrane (violet dying) were counted. The mean number of cells of each image was calculated for each group including the blank group, negative-siRNA group and siRNA-C group.

Statistical analysis. Data are presented as the mean \pm standard deviation. Each experiment was repeated three times at least. The results were analyzed using SPSS version 19.0 software (IBM Corp., Armonk, NY, USA). The significance of differences was determined using one-way analysis of variance with post hoc least significant difference, or Dunnett's t-test. $\mathrm{P}<0.05$ was considered to indicate a statistically significant difference.

\section{Results}

Optimization of the transfection sequence and concentration. Fig. 1A presents the flow cytometry results from cells transfected with FAM-labelled negative control siRNA at four different concentrations. The maximum transfection efficiency (88\%) was achieved at a concentration of $50 \mathrm{nmol} / \mathrm{l}$ (Fig. 1B). Fig. 1C presents the fluorescence and light microscopic images of the same field of view in cells transfected with $50 \mathrm{nmol} / \mathrm{l}$ siRNA.

Fig. 2A presents the $T K T$ expression levels at $48 \mathrm{~h}$ of cell transfected with the three $T K T$-specific siRNAs or negative control siRNA. Compared with negative control group, the knockdown efficiency of siRNA-C was the highest (TKT expression level, 0.35 relative to the negative control group). Fig. 2B presents the TKT expression levels at $72 \mathrm{~h}$ in cells transfected with various concentrations of siRNA-C. The results demonstrated that a concentration of $50 \mathrm{nmol} / 1$ achieved the greatest efficiency (TKT expression level, 0.21 relative to the negative control group), and this was in agreement with the flow cytometric analysis.

TKT knockdown decreases cell viability and induces cell cycle arrest. Fig. 3A presents the cell viability of A549 cells following a 72-h transfection. After $24 \mathrm{~h}$, the viability of cells transfected with siRNA-C was significantly reduced compared with the negative control group [OD values (mean \pm standard deviation) of siRNA-C group vs. negative control group were $0.2984 \pm 0.0371$ vs. $0.0952 \pm 0.0063(\mathrm{P}<0.0001)$ for $24 \mathrm{~h} ; 0.5582 \pm 0.0090$ vs. $0.1817 \pm 0.0037(\mathrm{P}<0.0001)$ for $48 \mathrm{~h}$; $1.3663 \pm 0.0833$ vs. $0.3519 \pm 0.0175(\mathrm{P}<0.0001)]$ for $72 \mathrm{~h}$. This 

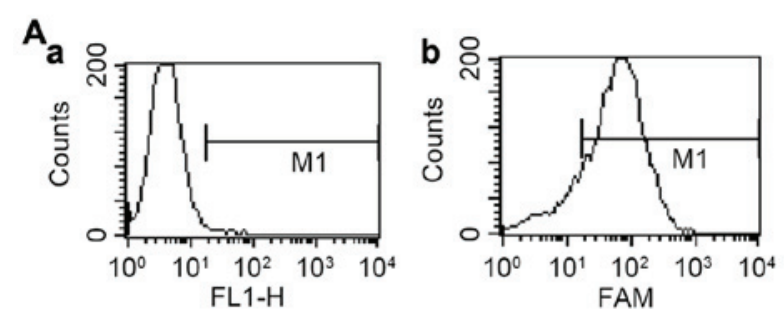

B
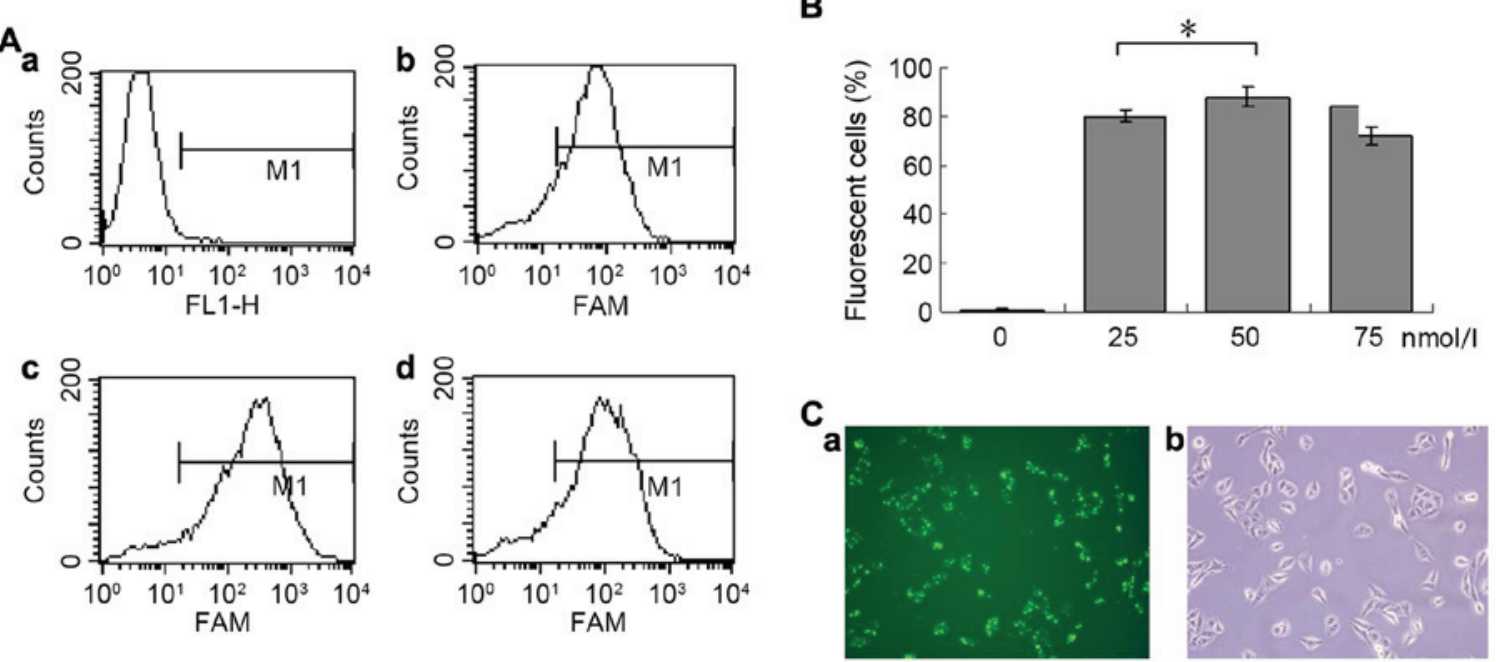

Figure 1. Optimization of the transfection concentration. (A) Four concentrations of FAM-labelled siRNA assessed by flow cytometry: (a) 0 , (b) 25 , (c) 50 and (d) $75 \mathrm{nmol} / \mathrm{l}$. (B) Percentage of fluorescent cells (M1) in the four concentration groups; a maximum transfection efficiency of $88 \pm 8 \%$ was achieved at $50 \mathrm{nmol} / 1$. (C) Microscopic images of cells transfected with $50 \mathrm{nmol} / 1$ siRNA (magnification, $\mathrm{x} 400$ ): (a) Fluorescence and (b) light microscopic images of the same field of view. "P<0.05. FAM, fluorescein amidite; siRNA, small interfering RNA; FL1-H, the cell stained by FAM.

indicated that downregulation of the TKT gene decreased the viability of A549 cells.

Fig. 3B presents the cell cycle distribution of the negative control and siRNA-C groups. The proportions of cells in $G_{1} / G_{0}$ phase in the negative control and siRNA-C groups were $56.51 \pm 2.0$ and $70 \pm 2.5 \%$, respectively $(\mathrm{P}=0.002)$. The proportions of cells in $\mathrm{G}_{2} / \mathrm{M}$ and $\mathrm{S}$ phases were $43.49 \pm 2.0$ and $30 \pm 2.5 \%(P=0.002)$, respectively. This suggests that the downregulation of TKT may arrest the cell cycle of A549 cells in $\mathrm{G}_{1} / \mathrm{G}_{0}$ phase.

TKT knockdown decreases cell migration. Compared with the negative control group, the wound healing ability of cells in the siRNA-C group decreased markedly (Fig. 4A); following a 24-h healing period, the wound sizes of the negative control vs. siRNA-C groups were $254.71 \pm 34.96$ vs. $349.12 \pm 37.43 \mu \mathrm{m}$ $(\mathrm{P}=0.0001)$. In the Transwell migration assay (Fig. 4B), the number of cells that migrated through the membrane was significantly decreased in the siRNA-C group compared with the negative control group $(150 \pm 49.0 /$ field vs. $250 \pm 47.8 /$ field, respectively; $\mathrm{P}<0.0001)$. This indicated that downregulation of TKT may inhibit cell migration in A549 lung cancer cells.

TKT knockdown decreases the mRNA expression levels of other metabolism-associated genes. As the key enzyme of the non-oxidative pathway, a decreased expression level of TKT can influence the expression levels of other key metabolic enzymes. The knockdown of TKT induced the downregulation of G6PDH, TAL, PRPS1, SORD and HK1 mRNA in A549 cells (Fig. 5).

Detection of TKT family genes. The human TKT gene family includes TKT, TKTL1 and TKTL2 (9). In the present study, the mRNA expression levels of TKTL1 and TKTL2 were detected in untreated A549 lung cancer cells using RT-qPCR. The results revealed that the Cq values of TKT, TKTL1-1, TKTL1-2, TKTL1-3 and TKTL2 were 20.73 $\pm 1.35,31.97 \pm 1.71$, $33.42 \pm 1.33,34.71 \pm 0.79$, respectively. This indicated that the
A

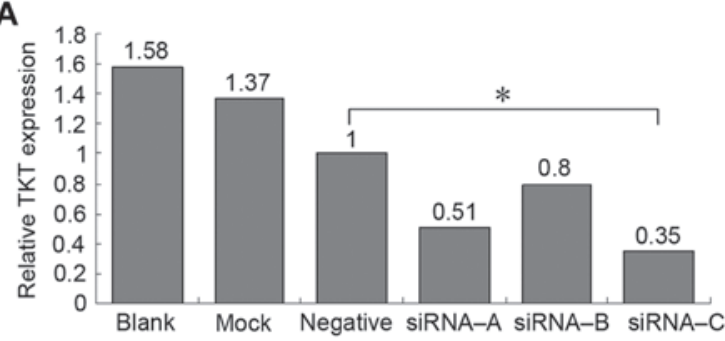

B

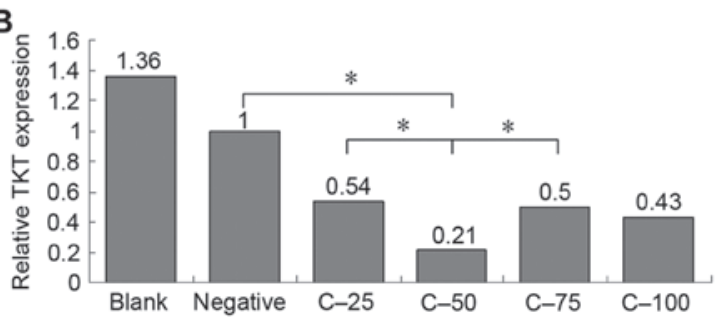

Figure 2. Selection of siRNA sequences for $T K T$ knockdown. (A) $T K T$ expression levels (relative to negative control) of cells transfected with the three $T K T$-specific siRNAs. (B) $T K T$ expression levels (relative to negative control) of cells transfected with TKT-siRNA-C at various concentrations (25-100 nmol/l). The TKT mRNA expression levels were measured using RT-qPCR. ${ }^{*} \mathrm{P}<0.05$. siRNA, small interfering RNA; TKT, transketolase; RT-qPCR, reverse transcriptase-quantitative polymerase chain reaction; C-25, 25 nmol/1 siRNA-C; C-50, 50 nmol/1 siRNA-C; C-75, 75 nmol/1 siRNA-C; C-100, 100 nmol/1 siRNA-C.

expression levels of TKTL1 and TKTL2 mRNAs are low or absent in these cells.

\section{Discussion}

The non-oxidative pathway mediated by TKT exploits the intermediates of glycolysis to synthesize ribose phosphate reversibly (6). A previous study confirmed that the non-oxidative pathway serves a vital role in malignant cell proliferation and metabolism (10). High activity and expression levels of TKT 
A

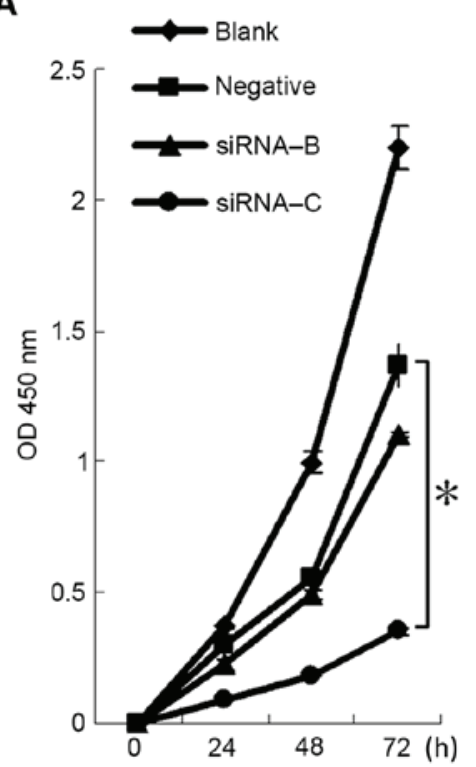

B
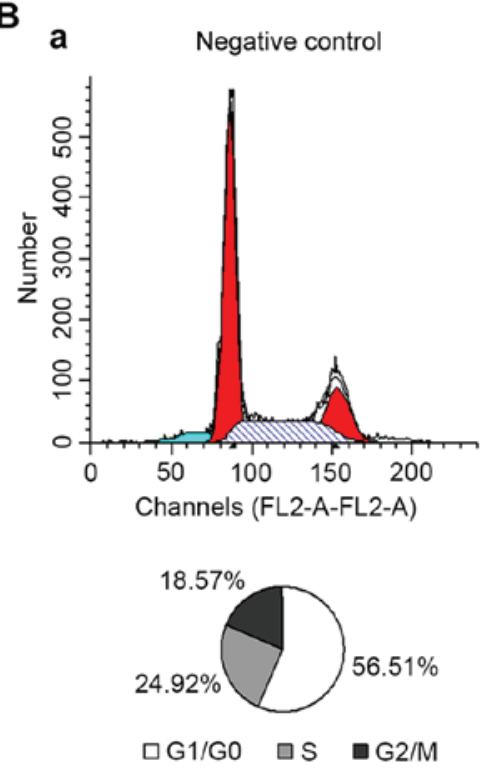

b SIRNA-C

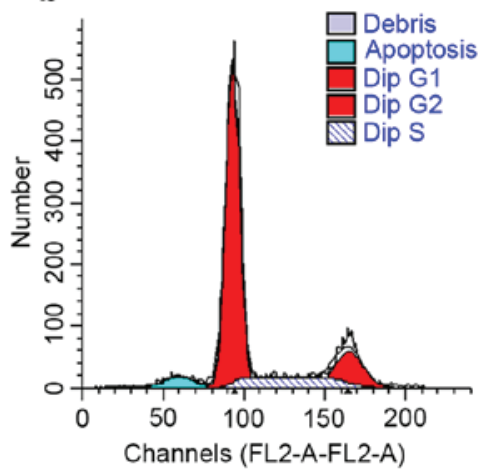

$14.36 \%$

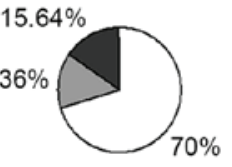

$\square G 1 / G 0 \quad \square S \quad \square G 2 / M$

Figure 3. Effect of TKT expression on cell viability and the cell cycle. (A) Cell viability (measured as OD) at 24, 48 and $72 \mathrm{~h}$ in cells transfected with siRNAs targeting TKT, and cells in the blank and negative control groups. (B) Cell cycle distribution in (a) cells transfected with negative control siRNA and (b) cells transfected with siRNA-C targeting TKT. "P<0.05. TKT, transketolase; OD, optical density; siRNA, small interfering RNA.

A

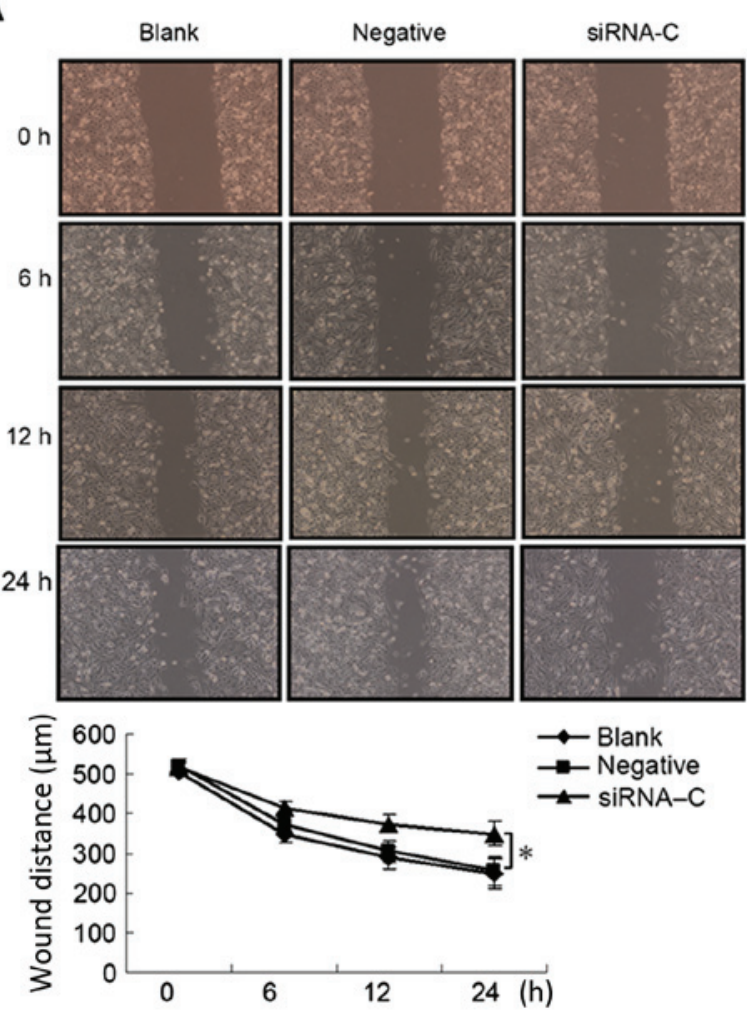

B

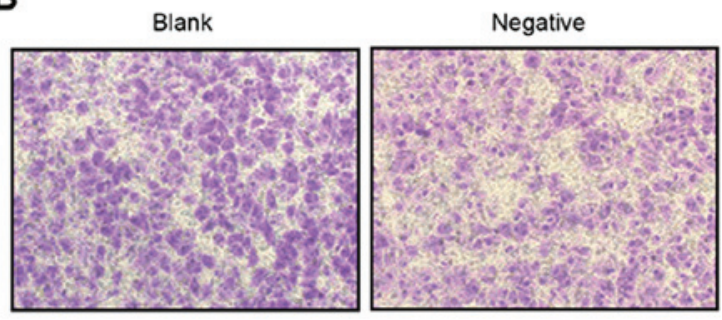

SIRNA-C
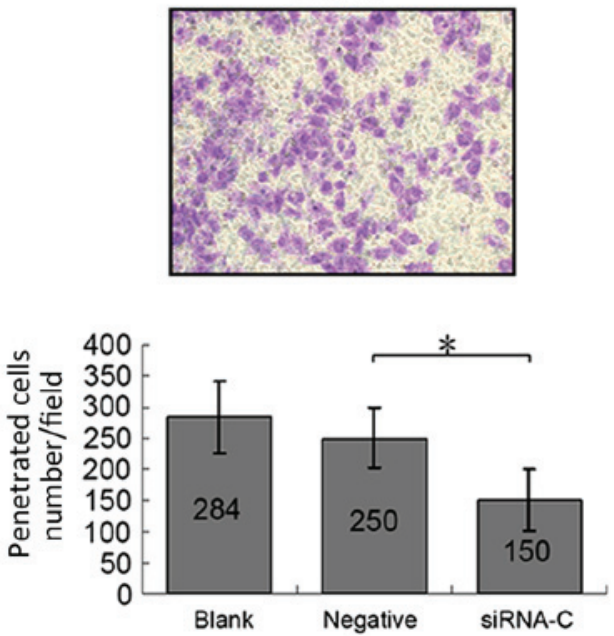

Figure 4. Representative images and quantified results of wound healing and Transwell migration assays. (A) Wound healing assay results at $0,6,12$ and $24 \mathrm{~h}$ in blank control, negative control and siRNA-C groups (magnification, x200). (B) Migrated cells on the membrane following Transwell migration assay in blank control, negative control and siRNA-C groups (magnification, $\mathrm{x} 400$ ). ${ }^{*} \mathrm{P}<0.05$. siRNA-C, small interfering RNA targeting transketolase.

have been identified in multiple tumor types (11). Furthermore, enhanced TKT activity may be a biomarker for tumor relapse and metastasis, indicating a poor prognosis (12).

The results of the present study indicated that the downregulation of TKT and decrease of ribose reduced A549 cell proliferation rate markedly, and arrested cell cycle at the $\mathrm{G}_{1} / \mathrm{G}_{0}$ phase. Targeting metabolic enzymes has great potential as an anticancer therapy. As a coenzyme of TKT, thiamine (vitamin B1) may be a target for tumor therapy, and its analogues, oxythiamine and pyrithiamine, have been demonstrated to 


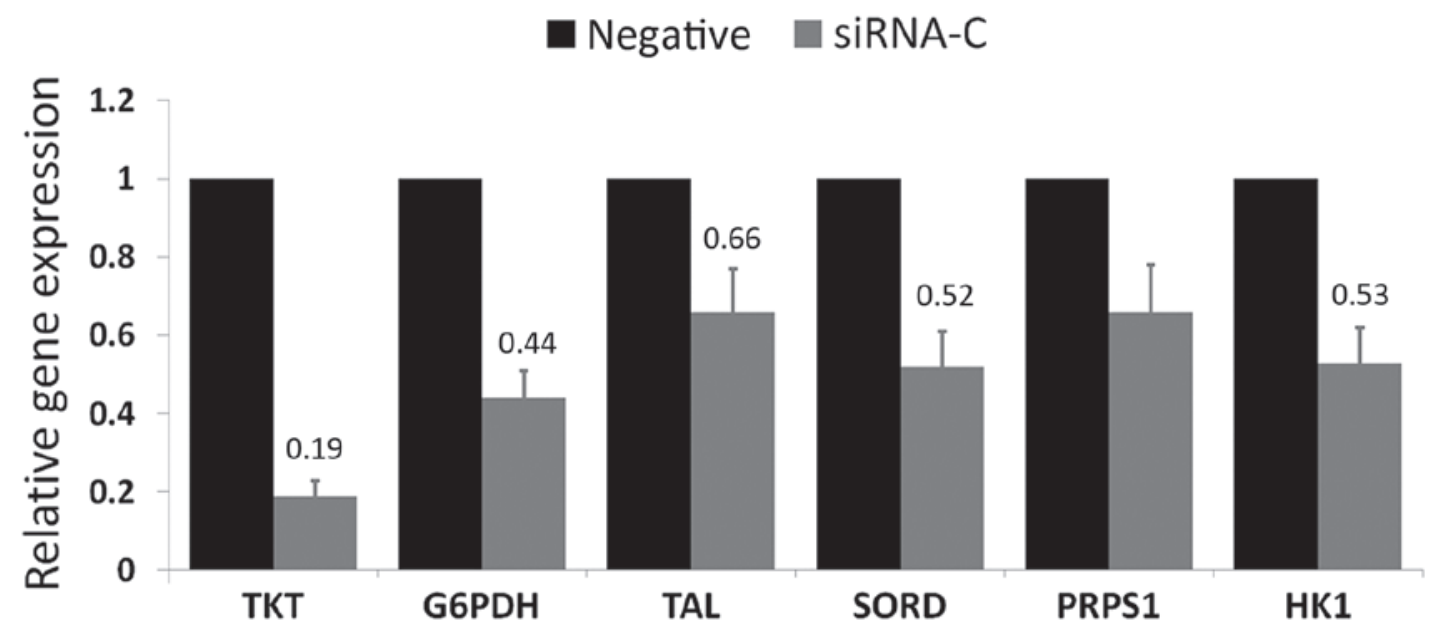

Figure 5. mRNA expression levels of $T K T$ and other key metabolic enzymes determined by reverse transcription-quantitative polymerase chain reaction. Relative to the negative control group (set as 1.0), the expression levels of TKT, G6PDH, TAL, SORD, PRPS1 and HK1 were 0.19, 0.44, 0.66, 0.52, 0.66 and 0.53 , respectively. Results are presented as the mean \pm standard deviation. Each sample was performed in triplicate. TKT, transketolase; TAL, transaldolase; G6PDH, glucose-6-phosphate dehydrogenase; PRPS1, phosphoribosyl pyrophosphate synthetase 1; SORD, sorbitol dehydrogenase; HK1, hexokinase 1.

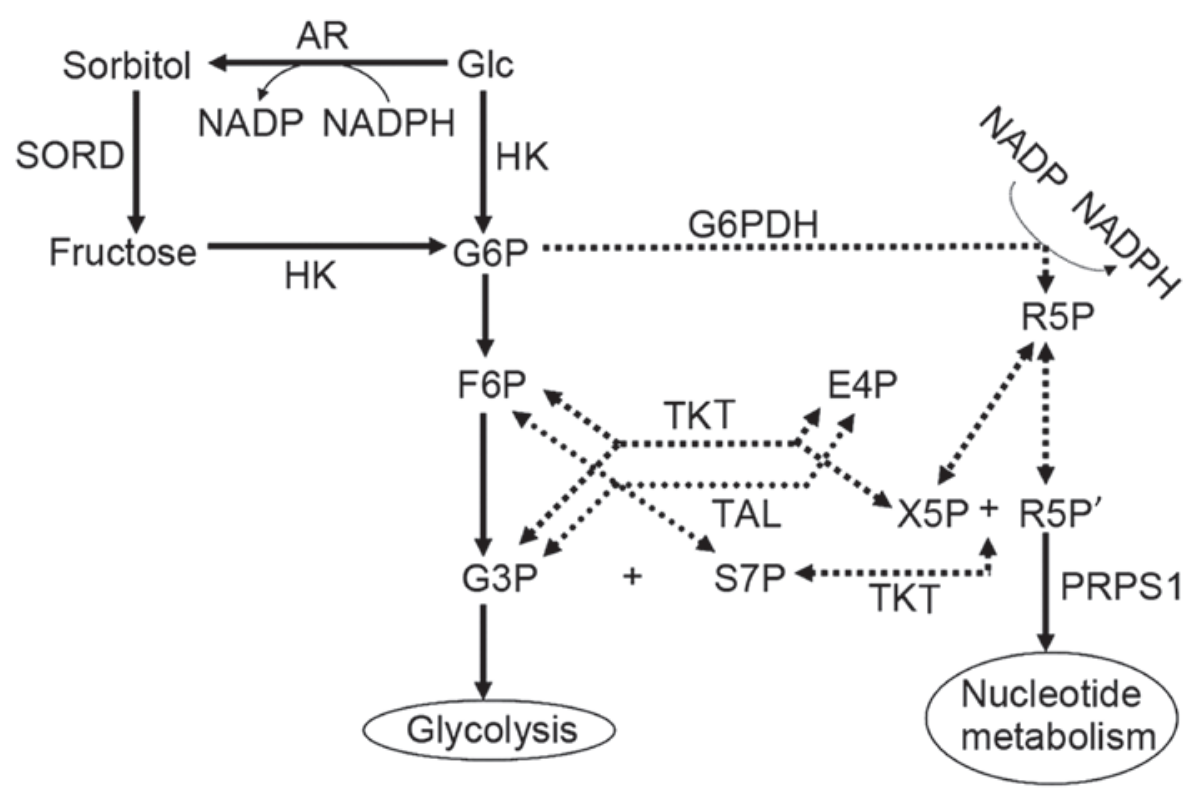

Figure 6. Pentose phosphate pathway (dotted lines) and associated metabolic pathways (solid lines). TKT, transketolase; TAL, transaldolase; G6PDH, glucose-6-phosphate dehydrogenase; HK, hexokinase; AR, aldose reductase; SORD, sorbitol dehydrogenase; Glc, glucose; G6P, glucose 6-phosphate; F6P, fructose 6-phosphate; R5P, ribose 5-phosphate; G3P, glyceraldehyde 3-phosphate; E4P, erythrose 4-phosphate; S7P, sedoheptulose 7-phosphate; X5P, xylulose 5-phosphate; R5P', ribulose 5-phosphate; NADP, nicotinamide-adenine dinucleotide phosphate; NADPH, reduced NADP; PRPS1, phosphoribosyl pyrophosphate synthetase 1 .

inhibit tumor growth significantly $(4,13)$. However, thiamine is also a coenzyme for pyruvate dehydrogenase and ketoglutarate dehydrogenase, which are key enzymes of the Krebs cycle. Furthermore, thiamine deficiency may affect thiamine-dependent enzymes dysfunction to various degrees and transketolase is the most vulnerable, induced by thiamine deficiency compared with other thiamine-dependent enzymes (pyruvate dehydrogenase and ketoglutarate dehydrogenase) (14). Previous studies have revealed that numerous natural compounds, including sugiol and epicatechin gallate, may inhibit cancer cell growth via the disturbance of TKT $(15,16)$. In the present study, TKT-siRNA inhibited A549 cell growth via $\mathrm{G}_{0} / \mathrm{G}_{1}$ cell cycle arrest, in agreement with previous experiments, and decreased the expression of G6PDH mRNA, which is the key enzyme of the oxidative signaling pathway. Jung et al (16) examined intracellular glutathione expression levels in TKT-knockdown cells, and revealed that glutathione levels were decreased, which induced reactive oxygen species production in DU145 cells. Thus, the presence of TKT was necessary for the production of the antioxidant enzyme NADPH and TKT knockdown increased reactive oxygen species generation, which may induce inflammation-injury (16).

$T K T$ is associated with numerous metabolic signaling pathways, including the polyol pathway, which includes SORD as its key enzyme (17). SORD expression was revealed to be decreased in TKT-knockdown cells in the present study. The 
polyol pathway provides substantial fructose for entry into the non-oxidative signaling pathway. When the non-oxidative pathway is inhibited, metabolites accumulate, fructose demand is reduced and SORD expression decreases. Fig. 6 presents the pentose phosphate pathway and its associated metabolic signaling pathways.

The human TKT gene family includes three members: TKT, TKTL1 and TKTL2. TKTL1 was considered to be a pseudogene until Coy et al (18) identified its encoded protein and named it in 2005; however, there are controversies over the results of previous studies on TKTL1 $(19,20)$, and whether TKTL1 can be regarded as a potential tumor marker requires further data for validation.

In conclusion, silencing TKT mRNA expression by RNAi can inhibit A549 lung cancer cell proliferation and cell migration, induce cell cycle arrest, and lead to the downregulation of other metabolic enzyme mRNAs. TKT may be a potential target for tumor therapy in the future. Further research investigating the mechanism underlying oncotherapy mediated by RNAi may provide a powerful tool for clinical application.

\section{Acknowledgements}

The present study was supported by a Basic Research Key Project from the Shanghai Science and Technology Commission (grant no. 12JC1402202) and the Key Project from the Shanghai Health Technical Committee (grant no. 201440041).

\section{References}

1. Torre LA, Bray F, Siegel RL, Ferlay J, Lortet-Tieulent J and Jemal A: Global cancer statistics, 2012. CA Cancer J Clin 65: 87-108, 2015.

2. Chen W, Zheng R, Zhang S, Zhao P, Zeng H and Zou X: Report of cancer incidence and mortality in China, 2010. Ann Transl Med 2: 61, 2014

3. Patra KC and Hay N: The pentose phosphate pathway and cancer. Trends Biochem Sci 39: 347-354, 2014.

4. Raïs B, Comin B, Puigjaner J, Brandes JL, Creppy E, Saboureau D, Ennamany R, Lee WN, Boros LG and Cascante M: Oxythiamine and dehydroepiandrosterone induce a G1 phase cycle arrest in Ehrlich's tumor cells through inhibition of the pentose cycle. FEBS Lett 456: 113-118, 1999.

5. Salas E, Roy S, Marsh T, Rubin B and Debnath J: Oxidative pentose phosphate pathway inhibition is a key determinant of antimalarial induced cancer cell death. Oncogene 35: 2913-2922, 2016.

6. Williams JF, Arora KK and Longenecker JP: The pentose pathway: A random harvest. Impediments which oppose acceptance of the classical (F-type) pentose cycle for liver, some neoplasms and photosynthetic tissue. The case for the L-type pentose pathway. Int J Biochem 19: 749-817, 1987.
7. Lu H, Lan WX, Bo L, Niu C, Zhou JJ and Zhu HL: Metabolic response of LLC xenografted mice to oxythiamine, as measured by [1H] NMR spectroscopy. Genet Mol Res 14: 11043-11051, 2015.

8. Fire A, Xu S, Montgomery MK, Kostas SA, Driver SE and Mello CC: Potent and specific genetic interference by double-stranded RNA in Caenorhabditis elegans. Nature 391: 806-811, 1998.

9. Schmittgen TD and Livak KJ: Analyzing real-time PCR data by the comparative C(T) method. Nat Protoc 3: 1101-1108, 2008.

10. Boros LG, Torday JS, Lim S, Bassilian S, Cascante M and Lee WN: Transforming growth factor beta2 promotes glucose carbon incorporation into nucleic acid ribose through the nonoxidative pentose cycle in lung epithelial carcinoma cells. Cancer Res 60: 1183-1185, 2000.

11. Lin CC, Chen LC, Tseng VS, Yan JJ, Lai WW, Su WP, Lin CH, Huang CY and Su WC: Malignant pleural effusion cells show aberrant glucose metabolism gene expression. Eur Respir J 37: 1453-1465, 2011.

12. Ricciardelli C, Lokman NA, Cheruvu S, Tan IA, Ween MP, Pyragius CE, Ruszkiewicz A, Hoffmann P and Oehler MK: Transketolase is upregulated in metastatic peritoneal implants and promotes ovarian cancer cell proliferation. Clin Exp Metastasis 32: 441-455, 2015

13. Yang CM, Liu YZ, Liao JW and Hu ML: The in vitro and in vivo anti-metastatic efficacy of oxythiamine and the possible mechanisms of action. Clin Exp Metastasis 27: 341-349, 2010.

14. Pekovich SR, Martin PR and Singleton CK: Thiamine deficiency decreases steady-state transketolase and pyruvate dehydrogenase but not alpha-ketoglutarate dehydrogenase mRNA levels in three human cell types. J Nutr 128: 683-687, 1998.

15. Sánchez-Tena S, Alcarraz-Vizán G, Marín S, Torres JL and Cascante M: Epicatechin gallate impairs colon cancer cell metabolic productivity. J Agric Food Chem 61: 4310-4317, 2013.

16. Jung SN, Shin DS, Kim HN, Jeon YJ, Yun J, Lee YJ, Kang JS, Han DC and Kwon BM: Sugiol inhibits STAT3 activity via regulation of transketolase and ROS-mediated ERK activation in DU145 prostate carcinoma cells. Biochem Pharmacol 97: 38-50, 2015.

17. Uzozie A, Nanni P, Staiano T, Grossmann J, Barkow-Oesterreicher S, Shay JW, Tiwari A, Buffoli F, Laczko E and Marra G: Sorbitol dehydrogenase overexpression and other aspects of dysregulated protein expression in human precancerous colorectal neoplasms: A quantitative proteomics study. Mol Cell Proteomics 13: 1198-1218, 2014.

18. Coy JF, Dressler D, Wilde J and Schubert P: Mutations in the transketolase-like gene TKTL1: Clinical implications for neurodegenerative diseases, diabetes and cancer. Clin Lab 51: 257-273, 2005.

19. Mayer A, Von Wallbrunn A and Vaupel P: Glucose metabolism of malignant cells is not regulated by transketolase-like (TKTL)-1. Int J Oncol 37: 265-271, 2010.

20. Hartmannsberger D, Mack B, Eggert C, Denzel S, Stepp H, Betz CS and Gires O: Transketolase-like protein 1 confers resistance to serum withdrawal in vitro. Cancer Lett 300: 20-29, 2011. 